\title{
Delay discounting and affective priming in individuals with negative schizotypy
}

\author{
Xin-lu Cai ${ }^{\text {ab, }, c}$, Michael Weigl ${ }^{\mathrm{d}}$, Bing-hui Liu ${ }^{\text {a,e }}$, Eric F.C. Cheung ${ }^{\mathrm{f}}$, Jin-hong Ding ${ }^{\text {g,* }}$, Raymond C.K. Chan ${ }^{\text {a,b,e,*** }}$ \\ a Neuropsychology and Applied Cognitive Neuroscience Laboratory, CAS Key Laboratory of Mental Health, Institute of Psychology, Beijing, 100101, PR China \\ b Sino-Danish College, University of Chinese Academy of Sciences, Beijing 100190, PR China \\ c Sino-Danish Center for Education and Research, Beijing 100190, PR China \\ d Experimental Neuropsychology Unit, Saarland University, Saarbrücken, Germany \\ e Department of Psychology, University of Chinese Academy of Sciences, Beijing 100190, PR China \\ ${ }^{\mathrm{f}}$ Castle Peak Hospital, Hong Kong, Special Administrative Region, PR China \\ ${ }^{g}$ Department of Psychology, Capital Normal University, Beijing 100037, PR China
}

\section{A R T I C L E I N F O}

Article history:

Received 19 June 2018

Received in revised form 8 December 2018

Accepted 20 December 2018

Available online $\mathrm{xxxx}$

\section{Keywords:}

Reward processing

Negative schizotypal personality

Anticipatory pleasure

\begin{abstract}
A B S T R A C T
Previous studies have demonstrated that patients with schizophrenia and individuals with schizotypy experience decreased anticipatory pleasure. However, it is unclear whether this decrease is contributed by altered reward processing at the proximal or distal future. In order to investigate the preference for receiving rewards in the proximal or distal future for individuals with schizophrenia spectrum disorders, individuals with either high or low levels of negative schizotypy performed a delay discounting task under positive, neutral and negative affective priming conditions. Compared with individuals with low levels of negative schizotypy, individuals with high levels of schizotypy exhibited increased delay discounting, preferring to choose immediate but smaller rewards instead of delayed but larger rewards across all three affective priming conditions. Negative affective priming elevated discounting for both groups compared with both the positive and neutral affective conditions. After dividing delayed temporal distance into the proximal and distal future, the results showed that individuals with high levels of negative schizotypy exhibited more preference for immediate but smaller rewards in the distal instead of proximal future compared with controls. Our results suggest that individuals with high levels of negative schizotypy have altered anticipatory reward processing, which is mainly attributed to alterations in representing rewards in the distal future. These findings extend the alterations in representing reward values from schizophrenia patients to schizotypal individuals, and suggest that diminished anticipatory pleasure in schizophrenia spectrum disorders may be due to changes in processing anticipatory rewards in the distal future.
\end{abstract}

(c) 2018 Published by Elsevier B.V.

\section{Introduction}

Reward processing is impaired in many mental disorders, including schizophrenia, major depressive disorder and autism spectrum disorders (Baskin-Sommers and Foti, 2015; Bottini, 2018; Yang et al., 2016). For schizophrenia spectrum disorders, previous studies have demonstrated that patients' reward processing is impaired at both the behavioural (Martinelli et al., 2018; Wang et al., 2015) and neural level relative to healthy individuals (Huang et al., 2016; Kirschner et al., 2015; Simon et al., 2015). More specifically, patients with schizophrenia appear to manifest the deficit in the anticipation of pleasure

\footnotetext{
* Correspondence to: J. Ding, 23 Baiduizi, Department of Psychology, Capital Normal University, Beijing 100037, PR China.

** Correspondence to: R.C.K. Chan, 16 Lincui Road, Institute of Psychology, Chinese Academy of Sciences, Beijing 100101, PR China.

E-mail addresses: dingjh@cnu.edu.cn (J. Ding), rckchan@psych.ac.cn (R.C.K. Chan).
}

rather than in the consummation of pleasure (Gard et al., 2007; Li et al., 2015; Lui et al., 2015; Mote et al., 2014), suggesting an abnormality in processing forthcoming rewards. Shankman et al. (2014) have proposed that anticipatory reward processing could be divided into reward processing in the proximal future and the distal future. This allows researchers to investigate whether the decreased anticipatory pleasure found in patients with schizophrenia is due more to alteration in processing of rewards in the proximal or distal future.

Delay discounting (temporal discounting) describes the decrease in subjective value of a future reward as a function of increasing delay (Mazur, 1987; Rachlin and Green, 1972) and has been shown to capture reward deficits in various psychiatric disorders (Imhoff et al., 2014; Story et al., 2016; Wilson et al., 2011). In the Delay Discounting Task, individuals are asked to decide between receiving small rewards immediately or larger rewards later (e.g. "Would you like to receive $\$ 10$ now or receive \$20 in 1 month?") to measure their intertemporal preference for receiving rewards (Green et al., 1996). Based on the hyperbolic model 
(Green et al., 1994), the delay discounting rate at different delayed time points can be calculated to assess reward preference at different temporal distances. A higher rate of delay discounting suggests that an individual prefers immediate rewards over delayed rewards and may be more impulsive (MacKillop et al., 2011; Odum, 2011). Previous neuroimaging studies have shown that the ventral striatum, the insula and the medial prefrontal cortex are involved in the reward processing of delay discounting tasks (Kable and Glimcher, 2007; Wittmann et al., 2010). By manipulating the temporal distance for delayed rewards, more involvement of the ventroanterior striatum and the nucleus accumbens has been shown to be correlated with longer delays (Steele et al., 2018; Wittmann et al., 2010). Moreover, activation of the insula and the striatum has been shown to hierarchically increase as delayed time increases (Tanaka et al., 2004).

Previous studies investigating delay discounting in patients with schizophrenia have produced inconsistent results. Some studies found that patients with schizophrenia exhibited greater delay discounting than healthy controls (Heerey et al., 2011; Yu et al., 2017; Weller et al., 2014), while others did not (Horan et al., 2017; MacKillop and Tidey, 2011; Wing et al., 2012). A functional magnetic resonance imaging (fMRI) study has shown that patients with schizophrenia had abnormal activation in the insula, the cingulate gyrus, the precuneus and the ventral striatum in a delay discounting task (Avsar et al., 2013). Among these regions, the changes of activation at the striatum and the insula, which have been shown to be correlated with temporal distances (Tanaka et al., 2004), indicates that there may be intertemporal decision-making changes as temporal distances vary in patients with schizophrenia. As for the inconsistent results regarding discounting rate in patients with schizophrenia, it remains unclear whether the abnormal discounting rate is due to the disease itself or other factors, such as smoking status, cognitive impairment, course of illness or medication.

Studying delay discounting in individuals with schizotypy may provide an alternative approach to minimize confounding factors. Schizotypy is a set of personality traits that convey vulnerability to schizophrenia (Meehl, 1962, 1989), and individuals with high levels of schizotypy have a higher risk of developing psychosis relative to individuals with low levels of schizotypy (Rawlings et al., 2008). Previous studies have found that individuals with high levels of schizotypy have impairments in cognition (Ettinger et al., 2015; Wang et al., 2008), emotion (Cohen et al., 2015; Wang et al., 2014), and social functioning (Wang et al., 2013). Furthermore, schizotypy could be divided into positive and negative schizotypy with different patterns of impairments (Ettinger et al., 2015; Yan et al., 2016). Previous studies have shown that individuals with elevated negative schizotypy experience decreased anticipatory pleasure (Engel et al., 2013; Loas et al., 2014; Martin et al., 2011), have deficits in emotion-related processing (Fung et al., 2017; Giakoumaki, 2016; Martin et al., 2011), and exhibit different neural responses when anticipating rewards (Yan et al., 2016) compared with controls. Interestingly, schizophrenia patients with negative symptoms also experience less anticipatory pleasure than patients without significant negative symptoms (Chan et al., 2010). Furthermore, in patients with recent-onset schizophrenia, more severe negative symptoms are associated with a lower capacity to anticipate pleasure (Mote et al., 2014). Thus, investigating anticipatory reward processing in individuals with negative schizotypy may provide a better understanding of anticipatory reward processing in schizophrenia, but few studies have investigated delay discounting in individuals with schizotypy.

Emotions have been shown to modulate intertemporal decisionmaking in the healthy population (Engelmann and Hare, 2016; Herman et al., 2018). Sohn et al. (2015) used affective pictures to induce positive, negative and neutral emotions and then asked participants to perform a delay discounting task. The participants showed higher discounting rate under both positive and negative arousal conditions compared with the neutral condition. Lerner et al. (2013) also found that participants were more impulsive under the negative emotion condition, but Luo et al. (2014) found that happy emotional primes increased impatience relative to fearful emotional primes. However, it is not clear whether such a modulatory effect of emotions on intertemporal reward processing exists or not in schizophrenia spectrum disorders. Impairments in emotion processing have been found in patients with schizophrenia spectrum disorders (Cohen et al., 2016; Giakoumaki, 2016), and patients with schizophrenia have also been found to experience more negative emotion (Cho et al., 2017). However, it is not known whether such impairments would alter reward processing or not. Therefore, investigating how emotions influence delay discounting in individuals with schizotypy may contribute to the understanding of the role that how emotions play in reward processing, and could facilitate the development of interventions to enhance reward processing in patients with schizophrenia spectrum disorders.

To address these questions, we examined and compared the delay discounting rate of individuals with high and low levels of negative schizotypy at the proximal and distal future using three delay discounting tasks under positive, neutral and negative affective priming conditions. Given that patients with schizophrenia exhibit greater delay discounting (Heerey et al., 2007; Weller et al., 2014), we hypothesized that individuals with high levels of negative schizotypy would choose smaller, immediate rewards rather than larger, delayed rewards and prefer rewards in the proximal future rather than the distal future. Since negative emotions increase delay discounting rate (Luo et al., 2014; Sohn et al., 2015) and patients with schizophrenia tend to experience more negative emotions (Cho et al., 2017), we further hypothesized that individuals with high levels of negative schizotypy would prefer immediate rewards in the positive or negative emotion conditions relative to the neutral affective condition compared with individuals with low levels of negative schizotypy.

\section{Method}

\subsection{Participants}

The revised Chinese version of the Chapman Social Anhedonia Scale (CSAS; Eckblad et al., 1982), which measures negative schizotypy (Chan et al., 2015), was used to define participants with high levels of negative schizotypy and participants with low levels of negative schizotypy. Three hundred and twenty-seven university students in Beijing were recruited to complete the CSAS. The mean score of the CSAS from this large sample pool was 10.28 ( \pm 5.61$)$. Participants whose scores were 1 SD higher than the mean were classified as individuals with high levels of negative schizotypy (CSAS scores $>15$ ) and participants whose scores were lower than the mean were classified as individuals with low levels of negative schizotypy (CSAS scores $<11$ ). The cut-offs were similar in our previous studies (Li et al., 2016a; Wang et al., 2016). Using this definition, 73 participants were recruited according to their scores on the CSAS in this study, 38 of whom had high levels of schizotypy and 35 had low levels of schizotypy. This study was approved by the Ethics Committee of the Institute of Psychology, the Chinese Academy of Sciences. Written informed consent was obtained from each participant.

\subsection{Stimulus material}

One hundred and twenty affective pictures were selected from the Chinese Affective Picture System (Lu et al., 2005). The pictures were categorized into three sets to be used in three emotional conditions: 40 pictures with positive valence and high arousal to elicit positive emotions, 40 pictures with negative valence and high arousal to elicit negative emotions, and 40 pictures with neutral valence and normal arousal corresponding to a neutral emotional state according to the norms of the Chinese Affective Picture System (valence: positive $=7.25 \pm 0.35$, negative $=1.97 \pm 0.47$, neutral $=4.40 \pm 0.35, F(2,117)=1784.10$, 
$p<0.001$; arousal: positive $=6.19 \pm 0.45$, negative $=6.77 \pm 0.36$, neutral $=3.88, F(2,117)=432.37, p<0.001)$.

\subsection{Delay discounting task}

A computerized delay discounting task was administered to all participants. In the task, participants were asked whether they preferred receiving 50 yuan immediately or 100 yuan at six delayed time points $(1,3,7,30,90$, or 180 days). They pressed the $F$ key or the $J$ key on the keyboard to choose the immediate or the delayed reward respectively. There was no time limit. Under each delay condition, the smaller value was changed based on the previous response until the preference changed: if a participant chose a delayed reward, the value of the immediate reward would increase by 5 yuan; and if a participant chose the immediate reward, the value of the immediate reward would decrease by 5 yuan. The indifference point was calculated by averaging the two immediate reward values before and after the preference changed in each delay.

\subsection{Other assessment}

Self-report questionnaires and IQ test were administrated to all participants. Self-report questionnaires included the Chinese version of the CSAS (Chan et al., 2012; Eckblad et al., 1982), the Beck Depression Inventory-I (BDI; Beck et al., 1988), and the adapted Chinese version of the Barratt Impulsiveness Scale Version 11 (BIS; Li et al., 2011; Patton et al., 1995).

Four subtests of the short-form of the Chinese version of the Wechsler Adult Intelligence Scale-Revised (WAIS-R) (information, similarity, arithmetic and digit span) were used to estimate the participants' IQ (Gong, 1992).

The Chinese version of the CSAS was employed to capture social anhedonia as the negative dimension of schizotypy. This version consists of 40 items and has been validated in previous studies (Chan et al., 2015; Chan et al., 2012). Higher scores indicate lower ability to experience pleasure from social activities.

The BDI was used to assess the severity of depressive symptoms. The $\mathrm{BDI}$ items were rated on a four-point scale to assess depressive symptoms of the participants. Higher scores indicate more severe depressive symptoms.

The adapted Chinese version of the BIS- 11 was used to measure impulsivity. The BIS contains three 10-item subscales: Non-planning Impulsiveness, Motor Impulsiveness and Attentional Impulsiveness. A higher total score indicates higher impulsivity.

\subsection{Procedure}

When participants arrived in the laboratory, they first completed the self-report questionnaires listed above. Next, a research assistant explained the Delay Discounting Task to the participants to ensure comprehension. Then, the participants began the task with practice trials where they had to choose between 1000 yuan and 2000 yuan in one week, three months or one year. When the participants were familiar with the procedure, they started the main experiment. Participants were instructed to complete the Delay Discounting Task under three emotional conditions: positive, negative and neutral, in a random order. In each emotional condition, participants were shown a set of 40 affective pictures to induce a positive, negative, or neutral emotional state before they began the task. In the Delay Discounting Task, the order of the delayed time points was also randomized. Before the experiments, participants were told that there were no right or wrong answers and they should make the choices they preferred. They were also told that they would receive a monetary reward which calculated based on their choices. The participants were paid 50 yuan/h for participating in the experiment and one of the indifference points was randomly chosen and multiplied by 0.1 as the additional compensation to enhance the salience of the task. After participants finished the experiment, their IQ was assessed by a trained research assistant. The whole experiment was run by E-Prime 2.0 (Psychology Software Tools, Inc., Pittsburgh, PA, USA).

\subsection{Data analysis}

For the delay discounting task, the Area Under the Curve (AUC) and the delay discounting rate $(k)$ were calculated (see Supplemental materials for details). Two kinds of delay discounting rates were calculated based on the hyperbolic model (Green \& Myerson, 2004; Mazur, 1987): the delay discounting rate at each delayed time point (calculated by the hyperbolic equation, referred as $k_{1}, k_{3}, k_{7}$, $k_{30}, k_{90}, k_{180}$ for each delay) and the delay discounting rate based on the whole six time points (using non-linear regression leastsquare analysis to determine the best fitting value $k_{\text {all }}$ ). Because the discounting rates were not normally distributed, we transformed $k$ to $\ln (k)$ for the subsequent analysis.

To increase the validity of the data analysis and the results for the delay discounting task, two widely used criteria (Horan et al., 2017; Tian et al., 2018; Wilson et al., 2011) were applied to identify unsystematic data (see Supplementary materials for details). Participants whose data under each emotional condition that met both criteria were considered unsystematic and excluded. Data from 12 participants (seven with high levels and five with low levels of negative schizotypy) were excluded as a result, leaving 31 participants in the high negative schizotypy group and 30 in the low negative schizotypy group.

Independent sample $t$-tests were used to compare age, IQ, scores on the CSAS, the BDI, the BIS and the three subscales of the BIS between the two groups. Chi-square tests were conducted to compare gender distribution and smoking status between groups. Correlation between the In $\left(k_{\text {all }}\right)$ and the AUC values in each emotional condition was calculated using Pearson correlation coefficients. The $\ln \left(k_{\text {all }}\right)$ and the AUC values were compared between the two groups using a $2 \times 3$ mixed multivariate analysis of variance (MANOVA) with group status as the betweensubject factor and affective priming (positive vs. negative vs. neutral) as the within-subject factor. Then, we defined the delay discounting rate based on different temporal distances (referred to as $k_{\text {tem }}$ ): the temporal distance was divided into the proximal future (1, 3 and 7 days) and the distal future (30, 90 and 180 days). The $k_{t e m}$ value for the proximal future was calculated by averaging $k_{1}, k_{3}, k_{7}$, and the $k_{\text {tem }}$ value for the distal future was calculated by averaging $k_{30}, k_{90}, k_{180}$. The $\ln \left(k_{t e m}\right)$ values were then entered into a $2 \times 2 \times 3$ mixed repeated-measures ANOVA with group status as the between-subject factor, temporal distance (proximal future vs. distal future) and emotional type (positive vs. negative vs. neutral) as within-subject factors. When main effects were significant in the ANOVA, post-hoc tests were conducted with Bonferroni corrections. When interaction effects were significant, the simple main effects test was conducted with Bonferroni corrections. Since impulsivity has been shown to be related to delay discounting (Ainslie, 1975; Hoe et al., 2018), scores on the BIS and the BDI were further entered into the MANOVA and the ANOVA as a covariate for further analysis independently. The $k_{\text {all }}$ values were calculated in MATLAB R2017b (MathWorks Inc.; https://www.mathworks.com). Other analyses were conducted using SPSS (Version 17, IBM).

\section{Results}

\subsection{Demographic information and group differences in the scale scores}

The two groups were matched for gender, age, IQ and smoking rate. There were significant differences between the two groups in the scores on the CSAS, the BDI, and the BIS (Table 1). 
Table 1

Demographic information for the high and low levels of schizotypal groups.

\begin{tabular}{|c|c|c|c|c|}
\hline & $\begin{array}{l}\text { High levels of negative schizotypal group } \\
\qquad(n=31) \\
\text { Mean (S.D.) }\end{array}$ & $\begin{array}{l}\text { Low levels of negative schizotypal group } \\
\qquad(n=30) \\
\text { Mean (S.D.) }\end{array}$ & $t / \chi^{2}$ & $p$ \\
\hline Age (years) & $19.77(0.990)$ & $19.767(1.104)$ & 0.028 & 0.978 \\
\hline Gender (male: female) & 14: 17 & $14: 16$ & 0.014 & 0.906 \\
\hline Smoking status (non-smoker: smoker) & $31: 0$ & $28: 2$ & 2.137 & 0.144 \\
\hline Education level (years) & $11.29(0.739)$ & $11.70(2.020)$ & -1.045 & 0.303 \\
\hline IQ & $118.16(9.744)$ & $118.13(8.241)$ & 0.012 & 0.990 \\
\hline CSAS & $20.32(4.736)$ & $5.83(3.761)$ & $13.205^{* *}$ & 0.000 \\
\hline BDI & $8.13(4.849)$ & $3.10(3.438)$ & $4.685^{* *}$ & 0.000 \\
\hline BIS-11 total & $39.19(8.828)$ & $32.14(9.468)$ & $3.011^{*}$ & 0.004 \\
\hline BIS-11 motor & $36.53(10.014)$ & $30.67(11.370)$ & $2.140^{*}$ & 0.036 \\
\hline BIS-11 attentional & $39.76(10.847)$ & $31.33(8.140)$ & $3.422^{*}$ & 0.001 \\
\hline BIS-11 nonplanning & $41.29(13.691)$ & 35.33 (12.675) & 1.762 & 0.083 \\
\hline
\end{tabular}

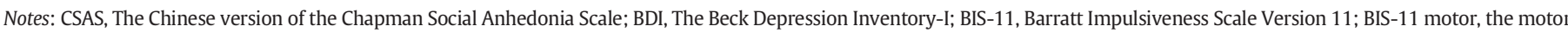

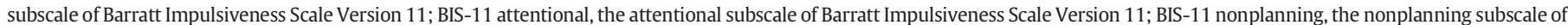
Barratt Impulsiveness Scale Version 11.

* $p<0.05$.

** $p<0.001$.

\subsection{Correlation for delay discounting parameters}

To provide evidence for the robustness of the parameters $\ln (k)$ and AUC (see Table 2 for descriptive statistics) of the delay discounting task, Pearson correlations were calculated showing that the $\ln \left(k_{\text {all }}\right)$ values were significantly and negatively correlated with the AUC values under each emotional condition for each group and across all participants (Table 3).

\subsection{Effects of affective priming and group status on delay discounting}

A 2 (group status: high vs. low levels of negative schizotypy) $\times 3$ (emotional type: positive vs. negative vs. neutral) MANOVA with repeated measures showed that the main effects for group and affective priming were both significant (group status: $F(2,58)=5.103, p=$ $0.009<0.05, \eta_{\mathrm{p}}^{2}=0.150$; affective priming: $F(4,56)=2.774, p=$ $\left.0.036<0.05, \eta_{\mathrm{p}}^{2}=0.165\right)$, but the interaction between groups status and affective priming was not significant $(F(4,56)=1.170, p=0.334$ $\left.>0.05, \eta_{\mathrm{p}}^{2}=0.077\right)$. Tests for univariate variables revealed significant group effects on the AUC values $(F(1,59)=8.453, p=0.005<0.05$, $\left.\eta_{\mathrm{p}}^{2}=0.125\right)$ (Fig. 1$)$ and $\ln \left(k_{\text {all }}\right)$ values $(F(1,59)=5.969, p=0.018<$ $0.05, \eta_{\mathrm{p}}^{2}=0.092$ ) (Fig. 2). There were also significant main effects for emotional types on both the AUC values $(F(2,58)=5.129, p=0.007$ $\left.<0.05, \eta_{\mathrm{p}}^{2}=0.080\right)$ and $\ln \left(k_{\text {all }}\right)$ values $(F(2,58)=5.592, p=0.005<$ $0.05, \eta_{\mathrm{p}}^{2}=0.087$ ). Post-hoc tests for the differences from the affective priming types showed that the $\ln \left(k_{\text {all }}\right)$ values were significantly higher and the AUC values were significantly lower under the negative affective priming condition $\left(\ln \left(k_{\text {all }}\right)\right.$ adjusted mean $=-3.52,95 \% \mathrm{C} \cdot \mathrm{I}$ : $[-4.14,-2.89]$; AUC adjusted mean $=0.48,95 \% \mathrm{C} \cdot \mathrm{I} .:[0.41,0.54]) \mathrm{com}-$ pared with the positive affective priming condition $\left(\ln \left(k_{\text {all }}\right): p=0.016\right.$; AUC: $p=0.024 ; \ln \left(k_{\text {all }}\right)$ adjusted mean $=-3.97,95 \%$ C.I.: $[-4.58$, $-3.35]$; AUC adjusted mean $=0.52,95 \%$ C.I.: $[0.46,0.59])$ and the neutral affective priming condition $\left(\ln \left(k_{\text {all }}\right): p=0.025\right.$; AUC: $p=0.020$; $\ln$ $\left(k_{\text {all }}\right)$ adjusted mean $=-3.95,95 \%$ C.I: $[-4.54,-3.37]$; AUC adjusted mean $=0.52,95 \%$ C.I.: $[0.45,0.59])$. The interaction between affective priming types and group status was not significant $\left(\ln \left(k_{\text {all }}\right): F(2,59)\right.$ $=1.740, p=0.180>0.05, \eta_{\mathrm{p}}^{2}=0.029$; AUC: $F(2,59)=1.874, p=$ $\left.0.158>0.05, \eta_{\mathrm{p}}^{2}=0.031\right)$. After adding BIS scores as a covariate, the results remained significant. After adding BDI scores as a covariate, the results remained significant as well. Overall, individuals with high levels of negative schizotypy showed significantly steeper discounting with lower AUC and higher $\ln \left(k_{\text {all }}\right)$ values compared with individuals with low levels of negative schizotypy and negative affective priming significantly increased delay discounting rates compared with positive and neutral affective priming.

\subsection{Effects of temporal distance and group status on delay discounting}

A 2 (group status: high vs. low levels of negative schizotypy) $\times 2$ (temporal distance: proximal vs. distal future) $\times 3$ (emotional type: positive vs. negative vs. neutral) ANOVA with repeated measures showed that there was a significant temporal distance effect on $\ln$ $\left(k_{\text {tem }}\right)\left(F(1,59)=123.98, p=0.000<0.05, \eta_{\mathrm{p}}^{2}=0.678\right)$, with a higher $k_{\text {tem }}$ in the proximal future. There was a significant effect for emotional types on $\ln \left(k_{\text {tem }}\right)\left(F(2,58)=8.63, p=0.001<0.05, \eta_{\mathrm{p}}^{2}=0.229\right)$, with a higher $\mathrm{k}$ under negative affective priming. The interaction between group status and temporal distance was also significant $(F(1,59)=$ 4.108, $p=0.047<0.05, \eta_{\mathrm{p}}^{2}=0.065$ ) (Fig. 3). The simple main effects test for the significant interaction showed that individuals with high levels of negative schizotypy had significantly higher $\ln \left(k_{\text {tem }}\right)$ values than individuals with low levels of negative schizotypy in the distal future $\left(F(1,59)=6.157, p=0.016<0.05, \eta_{\mathrm{p}}^{2}=0.094\right)$, but not in the proximal future $\left(F(1,59)=1.402, p=0.241>0.05, \eta_{p}^{2}=0.023\right)$. The results did not change after adding BIS scores as a covariate. After adding BDI scores as a covariate, the effect of temporal distance remained significant $\left(F(1,58)=41.14, p=0.000<0.05, \eta_{\mathrm{p}}^{2}=0.415\right)$, the interaction between group status and temporal distance became marginally significant $\left(F(1,58)=3.287, p=0.075<0.1, \eta_{\mathrm{p}}^{2}=0.054\right)$, but the effect of emotion became non-significant. Overall, the results

Table 2

Descriptive information for AUC and $k$.

\begin{tabular}{|c|c|c|c|c|c|c|}
\hline & \multicolumn{2}{|c|}{$\begin{array}{l}\text { High levels of negative schizotypal } \\
\text { Group } \\
\text { Mean (S.D.) }\end{array}$} & \multicolumn{2}{|c|}{$\begin{array}{l}\text { Low levels of negative schizotypal } \\
\text { Group } \\
\text { Mean (S.D.) }\end{array}$} & \multicolumn{2}{|c|}{$\begin{array}{l}\text { All participants } \\
\text { Mean (S.D.) }\end{array}$} \\
\hline & AUC & $\operatorname{Ln}\left(k_{\text {all }}\right)$ & AUC & $\operatorname{Ln}\left(k_{\text {all }}\right)$ & AUC & $\operatorname{Ln}\left(k_{\text {all }}\right)$ \\
\hline Positive emotion & $0.44(0.207)$ & $-3.30(1.903)$ & $0.61(0.317)$ & $-4.64(2.816)$ & $0.52(0.278)$ & $-3.95(2.470)$ \\
\hline Neutral emotion & $0.44(0.223)$ & $-3.36(1.901)$ & $0.60(0.295)$ & $-4.56(2.613)$ & $0.52(0.271)$ & $-3.94(2.338)$ \\
\hline Negative emotion & $0.36(0.178)$ & $-2.65(1.722)$ & $0.59(0.321)$ & $-4.39(3.014)$ & $0.47(0.280)$ & $-3.50(2.577)$ \\
\hline
\end{tabular}


Table 3

Correlation between AUC and $k$.

\begin{tabular}{cccc}
\hline & Group & $\mathrm{r}$ & $p$ \\
\hline \multirow{2}{*}{ Positive emotion } & High levels of negative schizotypal group & $-0.93^{* *}$ & 0.000 \\
& Low levels of negative schizotypal group & $-0.96^{* *}$ & 0.000 \\
& All participants & $-0.95^{* *}$ & 0.000 \\
Neutral emotion & High levels of negative schizotypal group & $-0.96^{* *}$ & 0.000 \\
& Low levels of negative schizotypal group & $-0.98^{* *}$ & 0.000 \\
& All participants & $-0.97^{* *}$ & 0.000 \\
Negative emotion & High levels of negative schizotypal group & $-0.83^{* *}$ & 0.000 \\
& Low levels of negative schizotypal group & $-0.96^{* *}$ & 0.000 \\
& All participants & $-0.94^{* *}$ & 0.000 \\
\hline
\end{tabular}

Notes: ${ }^{*} p<0.05 ;{ }^{* *} p<0.001$

showed that individuals with high levels of negative schizotypy had significantly higher delay discounting rate in the distal future than the proximal future compared with individuals with low levels of negative schizotypy.

\section{Discussion}

In the present study, we found that individuals with high levels of negative schizotypy had higher delay discounting rate and lower AUC values across all three emotional conditions, indicating that they showed greater preference for immediate rewards compared with individuals with low levels of negative schizotypy under each emotional condition. For the effect of affective priming, we observed that individuals preferred to choose immediate rewards in the negative affective priming condition compared with the positive and neutral affective priming conditions irrespective of the level of schizotypy. After dividing temporal distance, we found that individuals with high levels of negative schizotypy had higher delay discounting rate in the distal future, but not in the proximal future compared with individuals with low levels of negative schizotypy, indicating that individuals with high levels of negative schizotypy preferred to receive immediate but smaller rewards instead of delayed but larger rewards in the distal rather than proximal future. All the findings remained unchanged after adding BIS scores as a covariate, indicating that the devaluation of future rewards was not influenced by impulsivity. After adding BDI scores as a covariate, the effects of temporal distance and affective priming remained unchanged. The result that individuals with high levels of negative schizotypy exhibited increased delay discounting remained unchanged, but the preference to choose immediate rewards in the distal instead of proximal future became marginally significant, indicates that depressive symptoms may reduce such preference in individuals with high schizotypy.

Our finding of a steeper decrease in delay discounting in individuals with high levels of negative schizotypy replicates the findings from $\mathrm{Li}$ et al. (2016). They compared the delay discounting rate (from the Monetary-Choice Questionnaire) among participants with positive schizotypal personality features, participants with negative schizotypal personality features and controls, and found that participants with negative schizotypal personality features valued delayed rewards in the future less than the other two groups (Li et al., 2016b). The results from Li et al. (2016b) and the present study both suggest that negative schizotypy may be strongly associated with alterations in anticipatory reward processing. However, previous studies investigating delay discounting in patients with schizophrenia and their relatives have produced mixed results, with smoking status reported as a confounding factor (Heerey et al., 2011; Hoe et al., 2018; Yu et al., 2017; MacKillop and Tidey, 2011). In our study, we have carefully matched the smoking status between the two groups of participants to address this issue. Although smoking may influence reward pathways and may affect value representation (Barr et al., 2008; Bickel et al., 1999), the very low and matched smoking rate in our sample had minimized the effect of nicotine on the delay discounting task. Moreover, as an important phenotype of schizophrenia spectrum disorders (Meehl, 1989), the increased delay discounting rate in individuals with high levels of negative schizotypy suggests that altered intertemporal reward processing may already exist before illness onset and may not be a consequence of illness duration or medication. Instead, altered delay discounting may be considered a trait in individuals with schizophrenia spectrum disorders.

Gold et al. (2008) have proposed that patients with schizophrenia are impaired in reward value representation when processing rewards. Our findings have further extended this alteration into schizotypal individuals. Moreover, previous studies have demonstrated that individuals with schizotypy (Engel et al., 2013; Loas et al., 2014; Martin et al., 2011) and patients with schizophrenia (Li et al., 2015; Lui et al., 2015; Mote et al., 2014) experience less anticipatory pleasure compared with

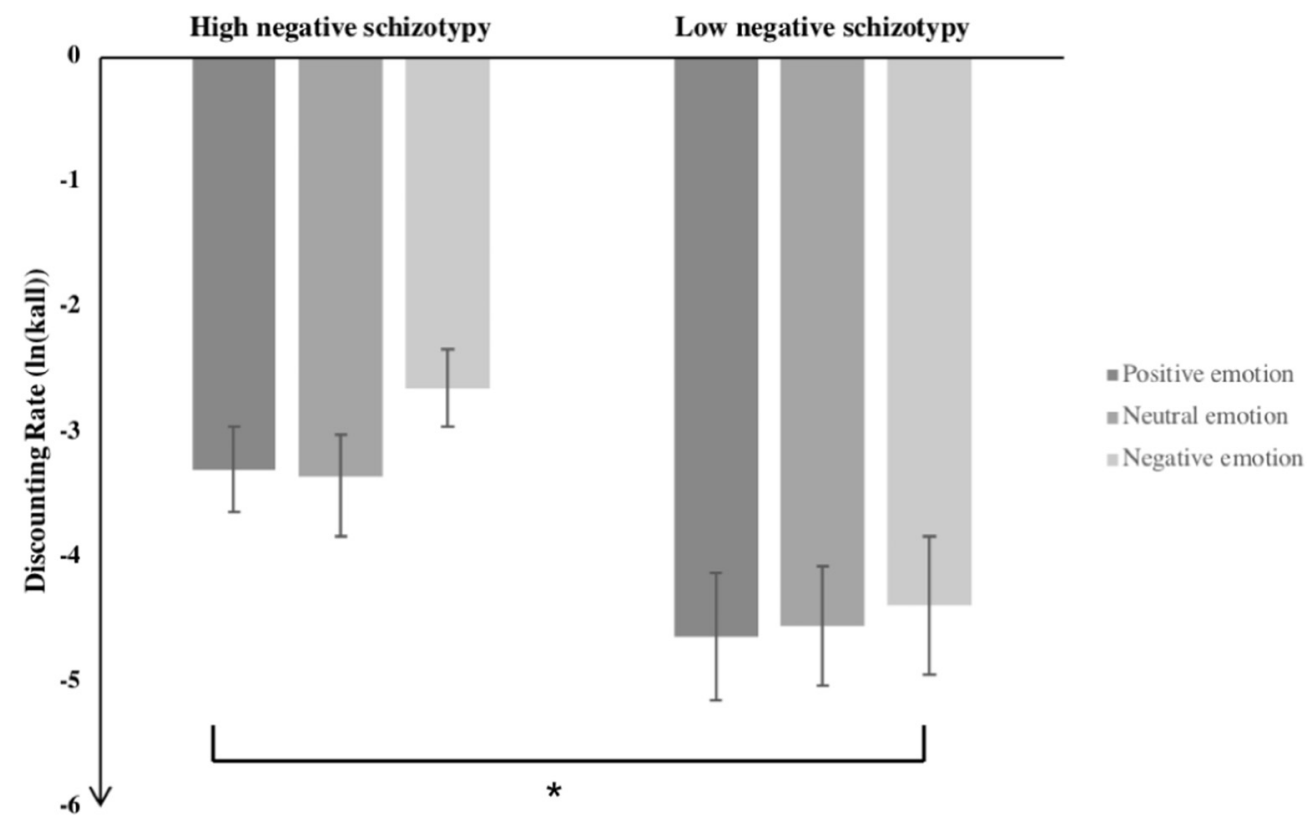

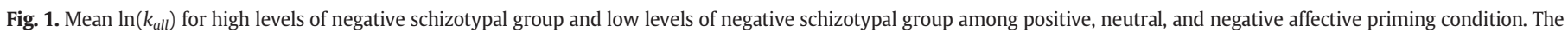
difference of $\ln \left(k_{\text {all }}\right)$ based on all emotion conditions between two groups was significant $(p<0.05)$. 


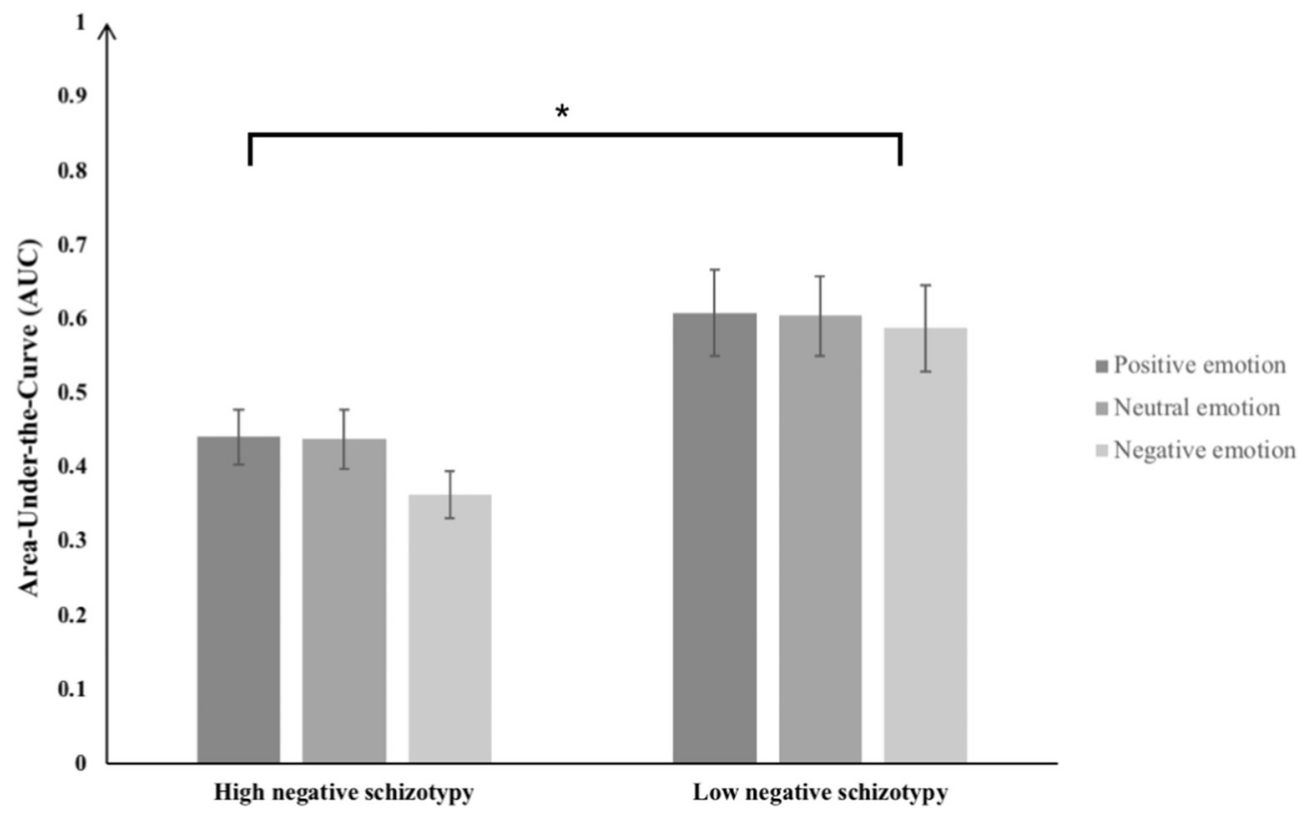

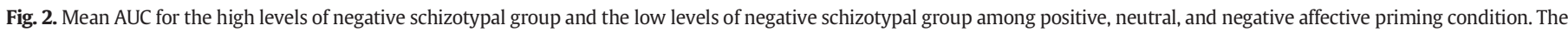
difference of AUC based on all emotion conditions between two groups was significant $(p<0.05)$.

controls. In our study, altered reward processing only appeared in the distal future instead of the proximal future for individuals with high levels of negative schizotypy, indicating that less anticipatory pleasure may mainly be due to altered reward processing in the distal future. A neuroimaging meta-analytic study has revealed that brain regions including the amygdala, the thalamus, the striatum and the insula play a key role in anticipating rewards (Oldham et al., 2018). In addition, a fMRI study has found that individuals with high levels of negative schizotypy exhibit diminished activation in the ventral striatum during reward anticipation (Yan et al., 2016). Moreover, the striatum is suggested to be associated with decision-making in different delayed time points (Steele et al., 2018; Wittmann et al., 2010). These converging lines of evidence indicate that the preference of rewards in the distal future in individuals with high negative schizotypy may be contributed by the altered activity in the striatum, which is important for motivational behaviour and negative symptoms in schizophrenia spectrum disorders. Another possible factor which may contribute to the preference in the distal future is prospective memory. Previous studies have found that episodic future thinking (prospection) reduces delay discounting in the healthy population, which implies less impulsivity in decision-making (Hu et al., 2016; Lin and Epstein, 2014). However, the impairments in prospective memory have been demonstrated in schizophrenia spectrum disorders, which may lead to more impulsive decision-making in individuals with high levels of negative schizotypy, manifesting as increased discounting rate in the distal rather than proximal future (Chen et al., 2016; Ordemann et al., 2014).

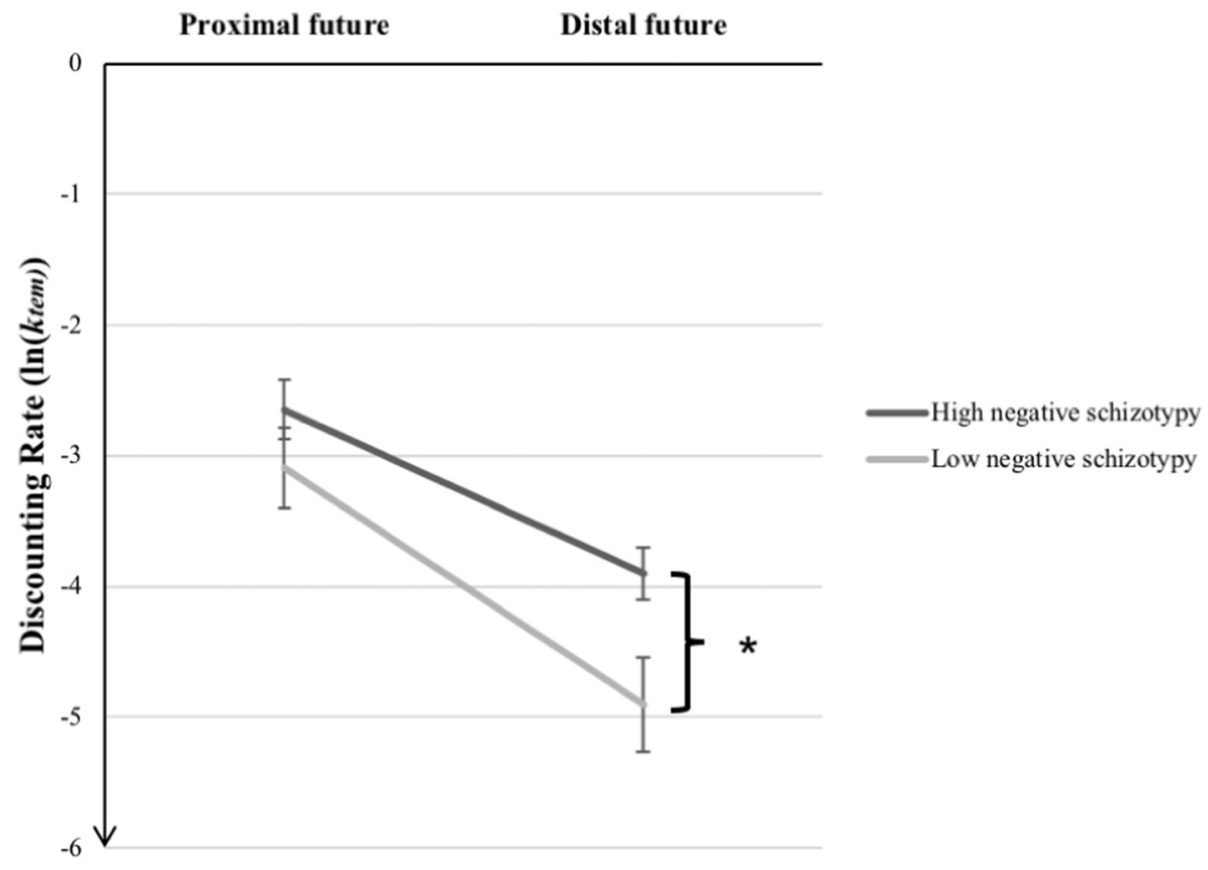

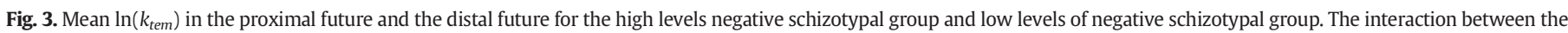
group status and the future temporal distance was significant $(p<0.05)$. 
For the effect of affective priming, the result that the negative affective condition increased delay discounting is in line with previous studies in the general population (Lerner et al., 2013; Sohn et al., 2015). In our study, the valence and the arousal of emotion induced by the affective pictures were similar as Sohn et al.'s study, supporting the validity of the affective pictures. Unexpectedly, there was no significant interaction between group status and affective priming types for the discounting rate, although previous studies have found that individuals with high levels of schizotypy have impaired emotion processing (Giakoumaki, 2016; Lui et al., 2016). This suggests that the effect of affective priming on decision-making is not significantly different between the two groups, which may be due to the limited magnitudes of elicited emotion and the small sample size.

Negative schizotypy was assessed based on social anhedonia in this study. Individuals with high levels of social anhedonia obtain diminished reward from social stimuli (Kwapil et al., 2009). The alterations in processing monetary rewards in this study suggest that individuals with high levels of negative schizotypy may not only be altered in processing social incentives but also monetary rewards. Both behavioural and neuroimaging findings have shown that individuals with social anhedonia exhibited specific deficits in social but not monetary incentives (Xie et al., 2014; Chan et al., 2016; Yan et al., 2016). The pattern of processing rewards from different domains in individuals with social anhedonia and the underlying neural mechanism warrants further investigation.

There are several limitations in this study. First, we failed to find a significant interaction between group status and affective priming types and we did not record the arousal and valence after participants were shown the affective pictures. Future studies should incorporate this step so that the subjective experience for the affective stimuli could be delineated. Secondly, we only defined temporal distance into the proximal and distal future. However, a more refined delineation of time span may be needed to examine more precisely how individuals evaluate rewards at different temporal distances in future studies. Thirdly, different samples from the schizophrenia spectrum, such as positive schizotypal individuals, first-degree relatives of schizophrenia patients, and schizophrenia patients, should be considered in future studies in exploring how delay discounting changes with the development of the disorder. Fourthly, although the participants were college students, it would be better to use the Mini-International Neuropsychiatric Interview (Sheehan et al., 1998) to exclude possible psychiatric disorders. Finally, incorporating neurophysiological and neuroimaging techniques in future studies could help to explore the underlying neurobiological mechanism of reward processing deficits.

\section{Conflict of interest}

None.

\section{Contributors}

Xin-lu Cai collected, analyzed and interpreted the data, and wrote up the first draft of the manuscript. Michael Weigl interpreted the findings and commented significantly to the revision of the manuscript. Bing-hui Liu helped to the data collection and contributed to the literature search. Eric Cheung and Jin-hong Ding commented significantly to the drafts of the manuscript. Raymond Chan designed the study, interpreted the findings and commented significantly to the drafts of the manuscript. All authors approved the final version for submission.

\section{Role of funding source}

The funding agents had no role in the study design, data collection, data analysis, interpretation of the findings, or the decision to publish the findings.

\section{Acknowledgements}

This work was supported by a grant from the National Natural Science Foundation of China (81571317), the Beijing Municipal Science and Technology Commission Grant (Z161100000216138), and the CAS Key Laboratory of Mental Health, Institute of Psychology.

\section{Appendix A. Supplementary materials}

Supplementary materials to this article can be found online at https://doi.org/10.1016/j.schres.2018.12.040.

\section{References}

Ainslie, G., 1975. Specious reward: a behavioral theory of impulsiveness and impulse control. Psychol. Bull. 82 (4), 463-496.

Avsar, K.B., Weller, R.E., Cox, J.E., Reid, M.A., White, D.M., Lahti, A.C., 2013. An fMRI investigation of delay discounting in patients with schizophrenia. Brain Behav. 3 (4), 384-401.

Barr, R.S., Pizzagalli, D.A., Culhane, M.A., Goff, D.C., Evins, A.E., 2008. A single dose of nicotine enhances reward responsiveness in nonsmokers: implications for development of dependence. Biol. Psychiatry 63 (11), 1061-1065.

Baskin-Sommers, A.R., Foti, D., 2015. Abnormal reward functioning across substance use disorders and major depressive disorder: considering reward as a transdiagnostic mechanism. Int. J. Psychophysiol. 98 (2), 227-239.

Beck, A.T., Steer, R.A., Carbin, M.G., 1988. Psychometric properties of the Beck depression inventory: twenty-five years of evaluation. Clin. Psychol. Rev. 8 (1), 77-100.

Bickel, W.K., Odum, A.L., Madden, G.J., 1999. Impulsivity and cigarette smoking: delay discounting in current, never, and ex-smokers. Psychopharmacology 146 (4), 447-454.

Bottini, S., 2018. Social reward processing in individuals with autism spectrum disorder: a systematic review of the social motivation hypothesis. Res. Autism Spectr. Disord. 45, $9-26$.

Chan, R.C., Wang, Y., Huang, J., Shi, Y., Wang, Y., Hong, X., Ma, Z., Li, Z., Lai, M.K., Kring, A.M., 2010. Anticipatory and consummatory components of the experience of pleasure in schizophrenia: cross-cultural validation and extension. Psychiatry Res. 175 (1-2), 181-183.

Chan, R.C., Wang, Y., Yan, C., Zhao, Q., McGrath, J., Hsi, X., Stone, W.S., 2012. A study of trait anhedonia in non-clinical Chinese samples: evidence from the Chapman Scales for Physical and Social Anhedonia. PLoS One 7 (4), e34275.

Chan, R.C., Shi, H.S., Geng, F.L., Liu, W.H., Yan, C., Wang, Y., Gooding, D.C., 2015. The Chapman psychosis-proneness scales: consistency across culture and time. Psychiatry Res. 228 (1), 143-149.

Chan, R.C., Li, Z., Li, K., Zeng, Y.W., Xie, W.Z., Yan, C., Cheung, E.F., Jin, Z., 2016. Distinct processing of social and monetary rewards in late adolescents with trait anhedonia. Neuropsychology 30 (3), 274-280.

Chen, X.J., Liu, LL, Cui, J.F., Wang Y. Chen, A.T. Li, F.H., Wang W.H., Zheng H.F., Gan, M.Y. Li, C.Q., Shum, D.H., Chan, R.C., 2016. Schizophrenia Spectrum disorders show reduced specificity and less positive events in mental time travel. Front. Psychol. 7, 1121.

Cho, H., Gonzalez, R., Lavaysse, L.M., Pence, S., Fulford, D., Gard, D.E., 2017. Do people with schizophrenia experience more negative emotion and less positive emotion in their daily lives? A meta-analysis of experience sampling studies. Schizophr. Res. 183, 49-55.

Cohen, A.S., Mohr, C., Ettinger, U., Chan, R.C.K., Park, S., 2015. Schizotypy as an organizing framework for social and affective sciences. Schizophr. Bull. 41, S427-S435.

Cohen, A.S., Callaway, D.A., Mitchell, K.R., Larsen, J.T., Strauss, G.P., 2016. A temporal examination of co-activated emotion valence networks in schizophrenia and schizotypy. Schizophr. Res. 170 (2-3), 322-329.

Eckblad, M., Chapman, L., Chapman, J., Mishlove, M., 1982. The Revised Social Anhedonia Scale (Unpublished test).

Engel, M., Fritzsche, A., Lincoln, T.M., 2013. Anticipatory pleasure and approach motivation in schizophrenia-like negative symptoms. Psychiatry Res. 210 (2), 422-426.

Engelmann, J., Hare, T., 2016. How Are Emotions Integrated into Choice?

Ettinger, U., Mohr, C., Gooding, D.C., Cohen, A.S., Rapp, A., Haenschel, C., Park, S., 2015. Cognition and brain function in schizotypy: a selective review. Schizophr. Bull. 41, S417-S426.

Fung, C.K., Moore, M.M., Karcher, N.R., Kerns, J.G., Martin, E.A., 2017. Emotional word usage in groups at risk for schizophrenia-spectrum disorders: an objective investigation of attention to emotion. Psychiatry Res. 252, 29-37.

Gard, D.E., Kring, A.M., Gard, M.G., Horan, W.P., Green, M.F., 2007. Anhedonia in schizophrenia: distinctions between anticipatory and consummatory pleasure. Schizophr. Res. 93 (1-3), 253-260.

Giakoumaki, S.G., 2016. Emotion processing deficits in the different dimensions of psychometric schizotypy. Scand. J. Psychol. 57 (3), 256-270.

Gold, J.M., Waltz, J.A., Prentice, K.J., Morris, S.E., Heerey, E.A., 2008. Reward processing in schizophrenia: a deficit in the representation of value. Schizophr. Bull. 34 (5), 835-847.

Gong, Y., 1992. Manual of Wechsler Adult Intelligence Scale-Chinese Version. Changsha: Chinese Map.

Green, L., Myerson, J., 2004. A discounting framework for choice with delayed and probabilistic rewards. Psychol. Bull. 130 (5), 769-792.

Green, L., Fristoe, N., Myerson, J., 1994. Temporal discounting and preference reversals in choice between delayed outcomes. Psychon. Bull. Rev. 1 (3), 383-389.

Green, L., Myerson, J., Lichtman, D., Rosen, S., Fry, A., 1996. Temporal discounting in choice between delayed rewards: the role of age and income. Psychol. Aging 11 (1), 79-84.

Heerey, E.A., Robinson, B.M., McMahon, R.P., Gold, J.M., 2007. Delay discounting in schizophrenia, Cogn. Neuropsychiatry 12 (3), 213-221.

Heerey, E.A., Matveeva, T.M., Gold, J.M., 2011. Imagining the future: degraded representations of future rewards and events in schizophrenia. J. Abnorm. Psychol. 120 (2), 483-489.

Herman, A.M., Critchley, H.D., Duka, T., 2018. The role of emotions and physiological arousal in modulating impulsive behaviour. Biol. Psychol. 133, 30-43.

Hoe, B.C., Barry, A.B., Koeppel, J.A., 2018. Impulsivity in unaffected adolescent biological relatives of schizophrenia patients. J. Psychiatr. Res. 97, 47-53.

Horan, W.P., Johnson, M.W., Green, M.F., 2017. Altered experiential, but not hypothetical, delay discounting in schizophrenia. J. Abnorm. Psychol. 126 (3), 301. 
Hu, X., Kleinschmidt, H., Martin, J.A., Han, Y., Thelen, M., Meiberth, D., Jessen, F., Weber, B., 2016. A reduction in delay discounting by using episodic future imagination and the association with episodic memory capacity. Front. Hum. Neurosci. 10, 663.

Huang, J., Yang, X.H., Lan, Y., Zhu, C.Y., Liu, X.Q., Wang, Y.F., Cheung, E.F.C., Xie, G.R., Chan, R.C.K., 2016. Neural substrates of the impaired effort expenditure decision making in schizophrenia. Neuropsychology 30 (6), 685-696.

Imhoff, S., Harris, M., Weiser, J., Reynolds, B., 2014. Delay discounting by depressed and non-depressed adolescent smokers and non-smokers. Drug Alcohol Depend. 135, $152-155$.

Kable, J.W., Glimcher, P.W., 2007. The neural correlates of subjective value during intertemporal choice. Nat. Neurosci. 10 (12), 1625-1633.

Kirschner, M., Hager, O.M., Bischof, M., Hartmann, M.N., Kluge, A., Seifritz, E., Tobler, P.N. Kaiser, S., 2015. Deficits in context dependent adaptive coding of reward values in schizophrenia. Eur. Neuropsychopharmacol. 25, S318.

Kwapil, T.R., Silvia, P.J., Myin-Germeys, I., Anderson, A.J., Coates, S.A., Brown, L.H., 2009. The social world of the socially anhedonic: exploring the daily ecology of asociality. J. Res. Pers. 43 (1), 103-106.

Lerner, J.S., Li, Y., Weber, E.U., 2013. The financial costs of sadness. Psychol. Sci. 24 (1), 72-79.

Li, X., Phillips, M., Xu, D., Zhang, Y., Yang, S., Tong, Y., Wang, Z., Niu, Y., 2011. Reliability and validity of an adapted Chinese version of Barratt impulsiveness scale. Chin. Ment. Health J. 25 (8), 610-615.

Li, Z., Lui, S.S.Y., Geng, F.L., Li, Y., Li, W.X., Wang, C.Y., Tan, S.P., Cheung, E.F.C., Kring, A.M., Chan, R.C.K., 2015. Experiential pleasure deficits in different stages of schizophrenia. Schizophr. Res. 166 (1-3), 98-103.

Li, X., Li, Z., Li, K., Zeng, Y.W., Shi, H.S., Xie, W.L., Yang, Z.Y., Lui, S.S., Cheung, E.F., Leung, A.W., Chan, R.C., 2016a. The neural transfer effect of working memory training to enhance hedonic processing in individuals with social anhedonia. Sci. Rep. 6, 35481.

Li, Z., Shi, Y.F., Parker, G.J., Huang, J., Yan, C., Lui, S.S.Y., Cheung, E.F.C., Neumann, D.L., Shum, D.H.K., Chan, R.C.K., 2016b. Devaluation of rewards for the future is associated with schizotypal personality features. Aust. Psychol. 51 (6), 481-489.

Lin, H., Epstein, L.H., 2014. Living in the moment: effects of time perspective and emotional valence of episodic thinking on delay discounting. Behav. Neurosci. 128 (1), 12-19.

Loas, G., Verrier, A., Monestes, J.L., 2014. Relationship between anticipatory, consummatory anhedonia and disorganization in schizotypy. BMC Psychiatry 14.

Lu, B., Hui, M., Yu-Xia, H., 2005. The Development of Native Chinese Affective Picture System-a Pretest in 46 College Students. Chinese Mental Health Journal.

Lui, S.S.Y., Wang, Y., Shi, Y.F., Au, A.C.W., Wong, P.T.Y., Chu, Z.L.S., Kring, A.M., Cheung, E.F.C., Chan, R.C.K., 2015. Subjective pleasure experience in patients with recentonset schizophrenia: a preliminary report. Psychiatry Res. 228 (1), 166-169.

Lui, S.S.Y., Shi, Y.F., Au, A.C.W., Li, Z., Tsui, C.F., Chan, C.K.Y., Leung, M.M.W., Wong, P.T.Y., Wang, Y., Yan, C., Heerey, E.A., Cheung, E.F.C., Chan, R.C.K., 2016. Affective experience and motivated behavior in schizophrenia Spectrum disorders: evidence from clinical and nonclinical samples. Neuropsychology 30 (6), 673-684.

Luo, S., Ainslie, G., Monterosso, J., 2014. The behavioral and neural effect of emotional primes on intertemporal decisions. Soc. Cogn. Affect. Neurosci. 9 (3), 283-291.

MacKillop, J., Tidey, J.W., 2011. Cigarette demand and delayed reward discounting in nicotine-dependent individuals with schizophrenia and controls: an initial study. Psychopharmacology 216 (1), 91-99.

MacKillop, J., Amlung, M.T., Few, L.R., Ray, L.A., Sweet, L.H., Munafo, M.R., 2011. Delayed reward discounting and addictive behavior: a meta-analysis. Psychopharmacology 216 (3), 305-321.

Martin, E.A., Becker, T.M., Cicero, D.C., Docherty, A.R., Kerns, J.G., 2011. Differential associations between schizotypy facets and emotion traits. Psychiatry Res. 187 (1-2), 94-99.

Martinelli, C., Rigoli, F., Dolan, R.J., Shergill, S.S., 2018. Decreased value-sensitivity in schizophrenia. Psychiatry Res. 259, 295-301.

Mazur, J.E., 1987. In: Commons, M.L., Mazur, J.E., Nevin, J.A. (Eds.), An Adjusting Procedure for Studying Delayed Reinforcement, pp. 55-73.

Meehl, P.E., 1962. Schizotaxia, schizotypy, schizophrenia. Am. Psychol. 17 (12), 827.

Meehl, P.E., 1989. Schizotaxia revisited. Arch. Gen. Psychiatry 46 (10), 935-944.

Mote, J., Minzenberg, M.J., Carter, C., Kring, A.M., 2014. Deficits in anticipatory but not consummatory pleasure in people with recent-onset schizophrenia spectrum disorders. Schizophr. Res. 159 (1), 76-79.

Odum, A.L., 2011. Delay discounting: trait variable? Behav. Process. 87 (1), 1-9.

Oldham, S., Murawski, C., Fornito, A., Youssef, G., Yucel, M., Lorenzetti, V., 2018. The anticipation and outcome phases of reward and loss processing: a neuroimaging metaanalysis of the monetary incentive delay task. Hum. Brain Mapp. 39 (8), 3398-3418.

Ordemann, G.J., Opper, J., Davalos, D., 2014. Prospective memory in schizophrenia: a review. Schizophr. Res. 155 (1-3), 77-89.

Patton, J.H., Stanford, M.S., Barratt, E.S., 1995. Factor structure of the Barratt impulsiveness scale. J. Clin. Psychol. 51 (6), 768-774.

Rachlin, H., Green, L., 1972. Commitment, choice and self-control. J. Exp. Anal. Behav. 17 (1), 15-+.
Rawlings, D., Williams, B., Haslam, N., Claridge, G., 2008. Is schizotypy taxonic? Response to Beauchaine, Lenzenweger, and Waller. Personal. Individ. Differ. 44 (8), 1663-1672.

Shankman, S.A., Katz, A.C., DeLizza, A.A., Sarapas, C., Gorka, S.M., Campbell, M.L., 2014. The different facets of anhedonia and their associations with different psychopathologies. Anhedonia: A Comprehensive Handbook. Vol. I. Springer, pp. 3-22.

Sheehan, D.V., Lecrubier, Y., Sheehan, K.H., Amorim, P., Janavs, J., Weiller, E., Hergueta, T. Baker, R., Dunbar, G.C., 1998. The Mini-International Neuropsychiatric Interview (M.I.N.I.): the development and validation of a structured diagnostic psychiatric interview for DSM-IV and ICD-10. J. Clin. Psychiatry 59 (Suppl. 20), 22.

Simon, J.J., Cordeiro, S.A., Weber, M.A., Friederich, H.C., Wolf, R.C., Weisbrod, M., Kaiser, S. 2015. Reward system dysfunction as a neural substrate of symptom expression across the general population and patients with schizophrenia. Schizophr. Bull. 41 (6), 1370-1378.

Sohn, J.H., Kim, H.E., Sohn, S., Seok, J.W., Choi, D., Watanuki, S., 2015. Effect of emotiona arousal on inter-temporal decision-making: an fMRI study. J. Physiol. Anthropol. 34.

Steele, C.C., Peterson, J.R., Marshall, A.T., Stuebing, S.L., Kirkpatrick, K., 2018. Nucleus accumbens core lesions induce sub-optimal choice and reduce sensitivity to magnitude and delay in impulsive choice tasks. Behav. Brain Res. 339, 28-38.

Story, G.W., Moutoussis, M., Dolan, R.J., 2016. A computational analysis of aberrant delay discounting in psychiatric disorders. Front. Psychol. 6.

Tanaka, S.C., Doya, K., Okada, G., Ueda, K., Okamoto, Y., Yamawaki, S., 2004. Prediction of immediate and future rewards differentially recruits cortico-basal ganglia loops. Nat. Neurosci. 7 (8), 887-893.

Tian, M., Tao, R., Zheng, Y., Zhang, H., Yang, G., Li, Q., Liu, X., 2018. Internet gaming disorder in adolescents is linked to delay discounting but not probability discounting. Comput. Hum. Behav. 80, 59-66.

Wang, Y., Chan, R.C.K., Yu, X., Shi, C., Cui, J.F., Deng, Y.Y., 2008. Prospective memory deficits in subjects with schizophrenia spectrum disorders: a comparison study with schizophrenic subjects, psychometrically defined schizotypal subjects, and healthy controls. Schizophr. Res. 106 (1), 70-80.

Wang, Y., Yeh, Y.H., Tsang, S.M., Liu, W.H., Shi, H.S., Li, Z., Shi, Y.F., Wang, Y., Wang, Y.N., Lui, S.S.Y., Neumann, D.L., Shum, D.H.K., Chan, R.C.K., 2013. Social functioning in Chinese college students with and without schizotypal personality traits: an exploratory study of the Chinese version of the first episode social functioning scale. PLoS One 8 (5).

Wang, Y., Lui, S.S., Zou, L.-q., Zhang, Q., Zhao, Q., Yan, C., Hong, X.-h., Tan, S.-p., Cheung, E.F. Chan, R.C., 2014. Individuals with psychometric schizotypy show similar social but not physical anhedonia to patients with schizophrenia. Psychiatry Res. 216 (2) 161-167.

Wang, J.A., Huang, J., Yang, X.H., Lui, S.S.Y., Cheung, E.F.C., Chan, R.C.K., 2015. Anhedonia in schizophrenia: deficits in both motivation and hedonic capacity. Schizophr. Res. 168 (1-2), 465-474.

Wang Y. Liu, W.H. Li, Z, Wei, X.H. Jiang X.Q Geng, F.L, Zou, L.Q Lui, S.S.Y., Cheung, E.F.C., Pantelis, C., Chan, R.C.K., 2016. Altered corticostriatal functional connectivity in individuals with high social anhedonia. Psychol. Med. 46 (1), 125-135.

Weller, R.E., Avsar, K.B., Cox, J.E., Reid, M.A., White, D.M., Lahti, A.C., 2014. Delay discounting and task performance consistency in patients with schizophrenia. Psychiatry Res. 215 (2), 286-293.

Wilson, V.B., Mitchell, S.H., Musser, E.D., Schmitt, C.F., Nigg, J.T., 2011. Delay discounting of reward in ADHD: application in young children. J. Child Psychol. Psychiatry 52 (3), 256-264.

Wing, V.C., Moss, T.G., Rabin, R.A., George, T.P., 2012. Effects of cigarette smoking status on delay discounting in schizophrenia and healthy controls. Addict. Behav. 37 (1), 67-72.

Wittmann, M., Lovero, K.L., Lane, S.D., Paulus, M.P., 2010. Now or later? Striatum and insula activation to immediate versus delayed rewards. J. Neurosci. Psychol. Econ. 3 15-26.

Xie, W.-z., Yan, C., Ying, X.-y., Zhu, S.-y., Shi, H.-s., Wang, Y., Cheung, E.F.C., Chan, R.C.K., 2014. Domain-specific hedonic deficits towards social affective but not monetary incentives in social anhedonia. Sci. Rep. 4, 4056.

Yan, C., Wang, Y., Su, L., Xu, T., Yin, D.Z., Fan, M.X., Deng, C.P., Wang, Z.X., Lui, S.S.Y., Cheung, E.F.C., Chan, R.C.K., 2016. Differential mesolimbic and prefrontal alterations during reward anticipation and consummation in positive and negative schizotypy. Psychiatry Res. Neuroimaging 254, 127-136.

Yang, X.H., Huang, J., Lan, Y., Zhu, C.Y., Liu, X.Q., Wang, Y.F., Cheung, E.F.C., Xie, G.R., Chan, R.C.K., 2016. Diminished caudate and superior temporal gyrus responses to effortbased decision making in patients with first-episode major depressive disorder. Prog. Neuropsychopharmacol. 64, 52-59.

Yu, L.Q., Lee, S., Katchmar, N., Satterthwaite, T.D., Kable, J.W., Wolf, D.H., 2017. Steeper discounting of delayed rewards in schizophrenia but not first-degree relatives. Psychiatry Res. 252, 303-309. 